\title{
Tumor-Infiltrating Lymphocytes (TILs) as a Biomarker of Abscopal Effect of Cryoablation in Breast Cancer: A Pilot Study
}

\author{
Sonia Y. Khan, BS ${ }^{1}$, Michael W. Melkus, $\mathbf{P h D}^{1}$, Fahmida Rasha, $\mathbf{P h D}^{2}$, Maribel Castro, $\mathbf{B S}^{\mathbf{1}}$, \\ Victoria Chu, MS ${ }^{1}$, Luis Brandi, $\mathrm{MD}^{3}$, Hafiz Khan, $\mathrm{PhD}^{4}$, Harvinder Singh Gill, $\mathrm{PhD}^{5}$, Kevin Pruitt, $\mathrm{PhD}^{2}$, \\ and Rakhshanda Layeequr Rahman, $\mathrm{MD}^{1,6}$ (1)
}

${ }^{1}$ Department of Surgery, School of Medicine, Texas Tech University Health Sciences Center, Lubbock, TX ; ${ }^{2}$ Department of Immunology and Molecular Microbiology, Texas Tech University Health Sciences Center, Lubbock, TX; ${ }^{3}$ Department of Pathology, School of Medicine, Texas Tech University Health Sciences Center, Lubbock, TX; ${ }^{4}$ Department of Public Health, Julia Jones Matthews, Texas Tech University Health Sciences Center, Lubbock, TX; ${ }^{5}$ Department of Chemical Engineering, Texas Tech University, Lubbock, TX ; ${ }^{6}$ Breast Center of Excellence, Texas Tech University Health Sciences Center, Lubbock, TX

\begin{abstract}
Background. Morphological evaluation of tumor-infiltrating lymphocytes (TILs) in breast cancer is gaining momentum as an immunological biomarker. This experiment evaluates the role of TILs in distant tumors as a measure of abscopal effect from cryoablation of breast cancer.

Methods. BALB/c mice underwent bilateral orthotopic transplant with 4T1-12B (triple-negative) cells. At 2 weeks, left tumors were treated by either resection (standard of care group) or cryoablation (intervention group) followed by resection of the distant right tumors 1 week posttreatment. TIL scores were calculated from hematoxylin and eosin-stained sections and phenotyped for cytotoxic T-lymphocyte (CTL) markers by immunofluorescence. Primarily resected tumors served as baseline $\left(\mathrm{T}_{\text {baseline }}\right)$, whereas resected distant right-sided served as
\end{abstract}

Sonia Y. Khan and Michael W. Melkus contributed equally to this work.

(C) The Author(s) 2022

First Received: 15 March 2021

Accepted: 14 November 2021;

Published Online: 29 January 2022

R. Layeequr Rahman, MD

e-mail: rakhshanda.rahman@ttuhsc.edu the readout for abscopal effect $\left(\mathrm{Abs}_{\text {Res }}\right.$ or $\left.\mathrm{Abs}_{\mathrm{Cryo}}\right)$. Mice were monitored for tumor recurrence and metastasis.

Results. The $\mathrm{Abs}_{\text {Cryo }}$ had a significant mean (SD) increase in stromal $(2.8[1.1] \% ; p=0.015)$ and invasive margin TILs (50 [12]\%; $p=0.02)$ compared with $\mathrm{T}_{\text {Baseline }}(1.0$ [0]\% and 31 [4.9]\%, respectively). CTL phenotyping revealed a significant increase in mean (SD) $\mathrm{CD} 8^{+} \mathrm{T}$ cells (15.7 [12.1]; $p=0.02$ ) and granzyme B (4.8 [3.6]; $p=$ 0.048) for the $\mathrm{Abs}_{\text {Cryo }}$ compared with $\mathrm{T}_{\text {Baseline }}$ (5.2 [4.7] and 2.4 [0.9], respectively). Posttreatment, the cryoablation group had no recurrence or metastasis, whereas the resected group showed local recurrence and lung metastasis in $40 \%$ of the mice. Postprocedure increase in TIL score of distant tumors was associated with decrease in tumor relapse $(p=0.02)$.

Conclusions. Cryoablation induced a robust tumor-specific TIL response compared with resection, suggesting an abscopal effect leading to the prevention of cancer recurrence and metastasis.

The concept of an immune-mediated response to target metastatic tumor cells located away from the locally treated tumor bed has existed for more than 60 years. While Mole coined the term "abscopal" ("ab" - away from, "scopus" - target) to describe this idea in regards to ionizing radiation, ${ }^{1}$ it is now applied to any form of truly local treatment, such as oncolytic virotherapy, ${ }^{2}$ histotripsy, ${ }^{3}$ and ablative techniques ${ }^{4,5}$ that result in a systemic immune 
response. Although uncommon, a 2015 study found 46 reported cases of the abscopal effect seen with local radiation therapy alone from 1969 to $2014 .{ }^{6}$ While attempts are still being made to elucidate the biological mechanism behind the abscopal effect, one popular theory regarding the abscopal effect seen with radiation lies with the liberation of tumor-associated antigens (TAAs) upon tumor stress or injury. ${ }^{7}$ A considerable boost in TAAs could stimulate a tumor-specific immune response. These TAAs would then be engulfed by antigen-presenting cells (APCs) and subsequently be presented to $\mathrm{CD} 8^{+} \mathrm{T}$ cells. ${ }^{8}$ Because the basis of the abscopal effect is an increase in immune response, it reflects a systemic link to local response.

Immune cell infiltration of tumors has been shown to provide predictive prognostic value in various tumor types, including lung, colon, ovarian, and breast cancers. ${ }^{9-12}$ In the case of breast cancer, tumor-infiltrating lymphocytes (TILs) have particular value. ${ }^{12}$ Murine and human studies have shown that most leukocyte subtypes predominantly contribute to either a protumor or antitumor microenvironment. For example, increased tumor infiltrating $\mathrm{CD} 8^{+} \mathrm{T}$ cells in breast cancer patients have antitumor activity and are associated with increased patient survival ${ }^{13}$ and response to chemotherapy. ${ }^{14}$ However, some T-cell subtypes, such as Th2 cells, have been associated with a decreased antitumor response. ${ }^{15}$ The effects of tumor-infiltrating B cells are not currently well known. ${ }^{16}$ Despite a lack of knowledge of the immune subpopulation comprising TILs, simple hematoxylin and eosin (H\&E)-stained tumor evaluation of the lymphocytic infiltration measured by TIL score can provide predictive and prognostic value in triple-negative breast cancer (TNBC) and human epidermal growth factor receptor 2 (HER2 ${ }^{+}$) breast cancer. ${ }^{12,17-20}$ TILs are an important component of the local tumor microenvironment. Because they have prognostic value as demonstrated by correlation with survival, ${ }^{17}$ and predictive value as demonstrated by correlation with pathological complete response to neoadjuvant chemotherapy, ${ }^{21,22}$ we sought to determine whether the TIL score of distant (untreated) tumors has value as a proxy measure of abscopal effect from the cryoablation-induced immune response in the primary tumor.

Cryoablation, unlike traditional resection, which removes the tumor completely, is a method of killing cancer cells via rapid freeze/thaw cycles while leaving the dead tumor cells in vivo. Cryoablation rapidly deep-freezes $\left(\leq-40{ }^{\circ} \mathrm{C}\right)$ the tumor, thus killing it while preserving potential TAAs, unlike other ablative techniques, such as thermal-heat ablation, which denatures tumor antigens. ${ }^{23}$ Because the ablated tumor remains in the patient, this introduces the potential for augmentation of an immune response to TAAs in vivo. ${ }^{24}$ Kumar et al. presented a case highlighting potential abscopal effect on axillary metastasis after cryoablation of breast cancer. ${ }^{25}$ However, there is no reliable way to measure the abscopal effect in the setting of early-stage disease (without demonstrable metastasis) where cryoablation is most effective.

In this experiment, we aimed to determine whether TILs in the distant tumor could be used as a biomarker for the abscopal effect following primary tumor treatment with cryoablation. We utilized a 2-tumor murine breast cancer model and compared TILs in H\&E-stained specimens of unmanipulated distant tumors after treatment of the primary tumors by either cryoablation or resection to measure abscopal effect.

\section{METHODS}

\section{Experimental Design: A Two Mammary Tumor Mouse Model to Evaluate the Abscopal Effect Post-Primary Tumor Treatment}

To address whether TILs in the distant tumor could serve as a biomarker of the abscopal effect resulting from local therapy of breast cancer, we used a 2-tumor mammary cancer mouse model. We utilized one tumor as a target for local therapy (either resection or cryoablation), followed by harvesting of the second, unmanipulated tumor to study the effect of the first treatment. A detailed experimental approach is described in Fig. 1. Local therapy represented two experimental arms: standard of care arm, and resection and intervention arm, i.e., cryoablation. Specimens collected were labeled according to their biological representation as follows:

$\mathrm{T}_{\text {Baseline }}-$ Treatment naïve tumor representing innate TIL response to tumor antigens.

$\mathrm{Abs}_{\text {Res }}$ - Distant tumor after local treatment of first tumor with resection representing TIL response affected by systemic effect of resection of first tumor.

$\mathrm{Abs}_{\text {Cryo }}$ - Distant tumor after local treatment of first tumor with cryoablation representing TIL response affected by systemic effect of cryoablation of first tumor.

\section{Cell Lines and Animals}

The mammary carcinoma 4T1-12B cell line (triplenegative) expresses luciferase for in vivo tracking of metastasis was obtained from TUFTS University (kindly provided by Sahagian et al). ${ }^{26}$ Cells were grown in Dulbecco's Modified Eagle's medium (DMEM) supplemented with $10 \%$ FBS plus $1 \%$ penicillin/streptomycin at $5 \% \mathrm{CO}_{2}$. Female BALB/c mice, aged 8-10 weeks, were purchased from Jackson Labs (Bar Harbor, ME). All work was conducted in accordance with TTUHSC-IACUC policies and approved protocol 17024 . 


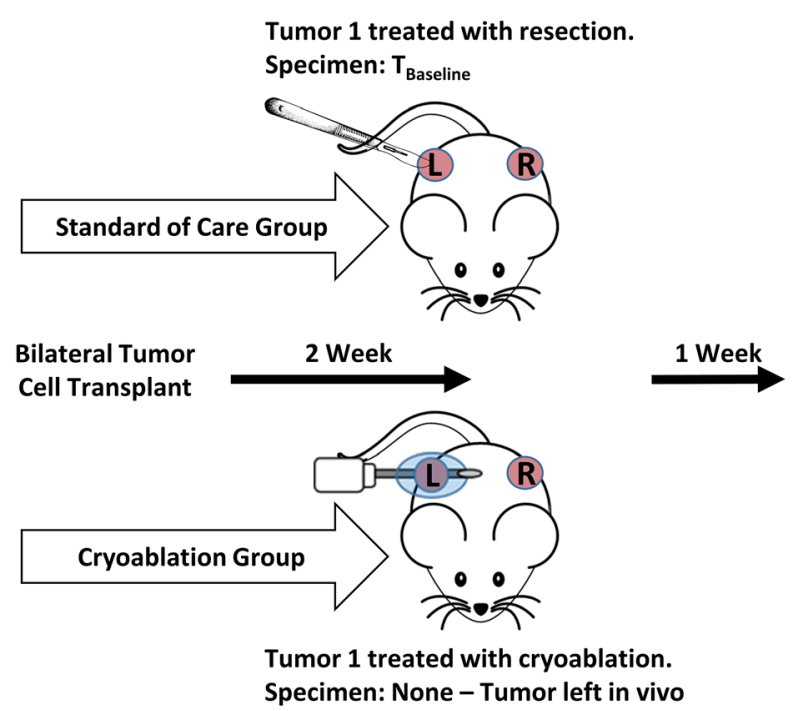

FIG. 1 Experimental approach. Mice were transplanted orthotopically with $1 \times 10^{6} 4 \mathrm{~T} 1-12 \mathrm{~B}$ cells in the mammary fat pad on each side to establish a 2-tumor breast cancer model to evaluate the abscopal effect post-tumor treatment comparing resection vs. cryoablation. At 2 weeks post-tumor cell transplant, the left tumor was treated either by resection or cryoablation. The resected tumor

\section{Tumor Cell Transplant}

4T1-12B cells (p22-p24) were passaged at 40-50\% confluency 24 hours prior to mouse transplant. Cells were isolated and resuspended at $2 \times 10^{7}$ cells $/ \mathrm{ml}$ in PBS. The mouse model was established by bilateral orthotopic injection with 4T1-12B $\left(1 \times 10^{6} /\right.$ side $)$ cells with a 27 -gauge needle into the mammary fat pad near nipple 4 and 9 regions.

\section{Monitoring Tumors}

Mice were observed three times per week for overall health and tumor growth. Tumors were monitored and volumes calculated using calipers and the formula $\mathrm{V}=$ $(\mathrm{W}(2) \times \mathrm{L}) / 2$. In vivo imaging (IVIS Lumina XR (Caliper Life Sciences, PerkinElmer ${ }^{\mathrm{TM}}$ ), mice were administered $100 \mu \mathrm{l}$ of luciferase substrate $(15 \mathrm{mg} / \mathrm{ml})$ intraperitoneally (IP) and imaged after 10 minutes under anesthesia with $2.5 \%$ isoflurane. Mice were imaged weekly for tumor growth, metastasis, and tumor recurrence.

\section{Surgical and Cryoablation Procedures}

All procedures were performed under strict aseptic technique. For mice in the standard of care group $(n=5)$, resections were performed with grossly negative surgical margins (Fig. 2A). The wound was then closed using 4-0 Prolene sutures (Ethicon, Somerville, NJ).
Tumor 2 (distant) resected for abscopal effect.

Specimen: Abs $_{\text {Res }}$
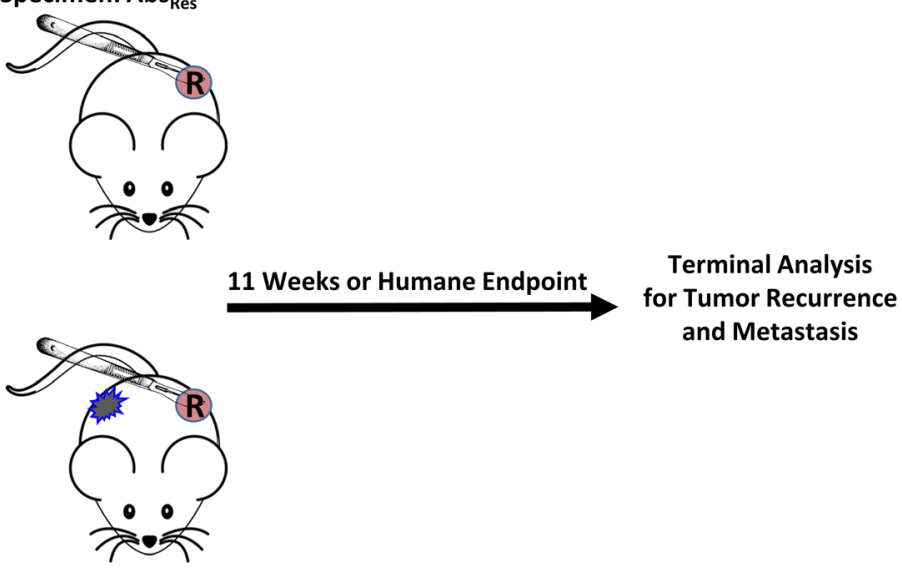

Tumor 2 (distant) resected for abscopal effect.

Specimen: Abs $_{\text {cryo }}$

was scored for TILs and served as the baseline control ( $\left.\mathrm{T}_{\text {Baseline }}\right)$. One week after treatment, the untreated right distant tumor was resected $\left(\mathrm{Abs}_{\text {Res }}\right.$ and $\left.\mathrm{Abs}_{\mathrm{Cryo}}\right)$ for TIL scores to measure the abscopal effect. Mice were then monitored for primary tumor recurrence and metastasis

For mice in the cryoablation group $(n=5)$, the skin was incised and retracted away from tumor with stay sutures to prevent frostbite. Cryoablation was performed using the liquid nitrogen-based Visica ${ }^{\circledR} 2$ Treatment System (Sanarus Technologies Inc., Pleasanton, CA) (Fig. 2B). The cryoprobe was placed directly on the tumor mass, and cryoablation was performed at a high rate of freeze with each tumor undergoing a freeze/thaw/freeze cycle. After completion of the cryoablation, the tumor was allowed to thaw and skin was closed over the tumor.

After local therapy (surgical resection or cryoablation), mice were administered $0.05 \mathrm{mg} / \mathrm{kg}$ of buprenorphine (Par Pharmaceuticals, Chestnut Ridge, NY) intraperitoneally in $200 \mu \mathrm{l}$ of saline. Mice were provided MediGel Hazelnut (Clear $\mathrm{H}_{2} \mathrm{O}^{\circledR}$, Portland, ME) supplemented with $0.5 \mathrm{mg} / \mathrm{ml}$ of Rimadyl (Zoetis, Parsippany, NJ) for pain management and monitored for 5 days for postsurgical/cryoablation complications.

\section{Pathology}

Standard H\&E staining of paraffin-embedded tissue was used for histopathological examination of primary and metastatic tumors. Sections were examined and photographed using a Leica Model DM 2000 LED microscope (Buffalo Grove, IL) equipped with an MC 170 HD camera and Leica Application Suite version 3.4 software. 
FIG. 2 Tumor treatment procedures. A Left, tumor growth in mammary fat pad (red arrow); right, resected mammary tumor (serves as baseline control). B Left, cryoablation technique freezing the tumor; right, completely frozen tumor - remains in the mouse. C IVIS analysis showing tumors before and posttreatment. Both resection and cryoablation treatments of the left tumor showed no luciferase activity 24 hours posttreatment
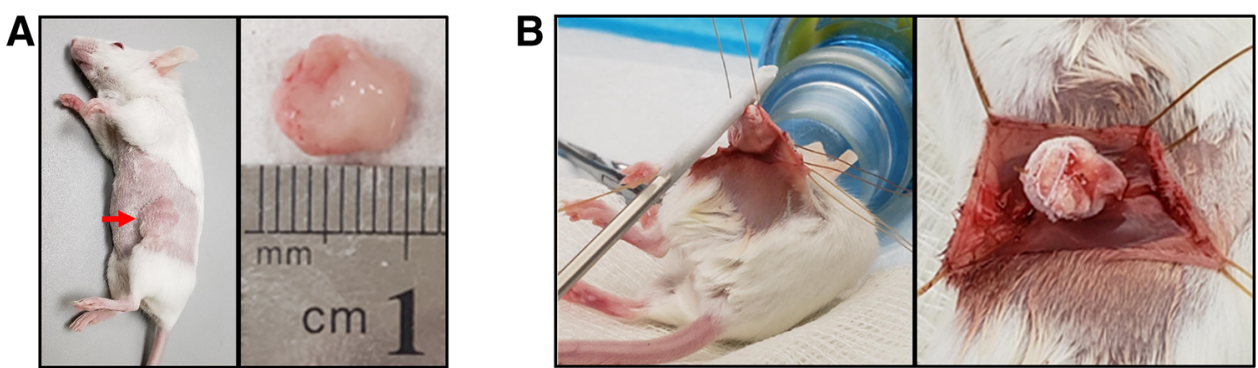

C
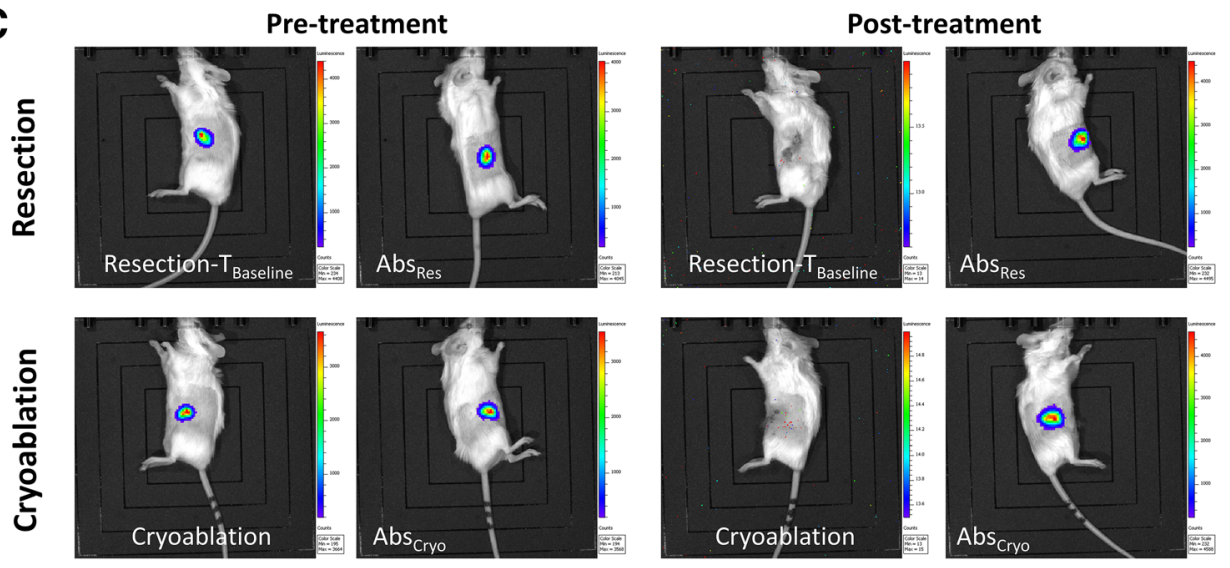

\section{TIL Scoring}

The evaluation of TILs was based on the guidelines set forth by the International TILs Working Group $2014^{17}$ and updates from the International Immuno-Oncology Biomarker Working Group on Breast Cancer. ${ }^{21}$ For this study, we analyzed stromal, intratumor, and invasive margin TILs in all tumors to gather the broadest scope of data for correlations between tumor treatment and abscopal effect.

To analyze TILs, an appropriate area was selected that reflected the immune infiltrate present throughout the entire specimen. Once a representative area was found at a low magnification, the magnification was then increased to $\times 200-400$ to determine the types of inflammatory infiltrate and provide more accurate scoring. TIL scoring was determined by using the area occupied by TILs as the numerator and the local tissue in the field as the denominator. This was repeated with three representative areas. Then, the TIL score was averaged to provide the most accurate score for the specimen.

Stromal TILs are dispersed lymphocytes that are located within the tumor border but do not make direct contact with any tumor cells. Intratumor TILs have intimate cell-to-cell contact with tumor cells with no stromal separation. Invasive margin TILs are similar to stromal; however, they are in the peripheral region of the tumor.

To provide the most accuracy, each of the 3 categories (stromal, intratumor, and invasive margin) of the 15 specimens $\left(5 \mathrm{~T}_{\text {Baseline, }} 5 \mathrm{Abs}_{\text {Res }}\right.$, and $\left.5 \mathrm{Abs}_{\text {Cryo }}\right)$ were independently scored by two trained professionals and then averaged. During analysis, necrotic areas, crush artifacts, healthy tissue, fibrous areas, and focusing on hotspots were avoided.

\section{Immunofluorescence Staining}

TILs also were quantitated for cytotoxic $\mathrm{T}$ cells by immunofluorescence staining with biomarkers for CD3e, $\mathrm{CD} 8 \alpha$, and granzyme B. Tumor sections were deparaffinized, underwent heat-induced antigen retrieval, and were incubated overnight at $4{ }^{\circ} \mathrm{C}$ with rabbit polyclonal antibodies CD3 $\varepsilon$ (D4V8L; Cell Signaling) at 1:100, CD8 $\alpha$ (D4W2Z; Cell Signaling) at 1:100, or granzyme B (EPR22645-206; Abcam Inc) at 1:200 dilution in blocking buffer (PBS, 0.1\% Saponin, 5\% BSA). Slides were washed with PBS and incubated with an Alexa Fluor 568-conjugated secondary goat anti-rabbit IgG (A11036, Thermo Fisher Scientific) at 1:300 dilution, washed, and mounted with Prolong ${ }^{\mathrm{TM}}$ Gold with DAPI (Invitrogen). Image collection and analysis were performed under blinded conditions. Samples were imaged using a confocal microscope Nikon T-1E with a 40x objective and NIS software then analyzed using AdipoGauge software for Windows (Version 2.0). ${ }^{27}$ Antibody fluorescence was normalized using TRITC/DAPI staining for each sample with the lowest reading for each antibody $(\mathrm{CD} 3 \varepsilon, \mathrm{CD} 8 \alpha$, granzyme $\mathrm{B})$ in the baseline group being set at 1 and the other samples calculated as fold change. 


\section{Necropsy}

Mice were euthanized using $\mathrm{CO}_{2}$ followed by exsanguination and cardiac puncture according to AVMA Guidelines for the Euthanasia of Animals: 2020 Edition. Animals necropsied with the following tissues collected for analysis: mammary tumors, spleens, liver, lungs, femurs, kidneys, heart, and spines. Tumor volume was calculated using calipers measurements. All tissues were visually examined for metastasis and the liver, lungs, spleen, and tumors were analyzed with histopathology by H\&E.

\section{Statistics}

All statistical analyses were performed using GraphPad Prism version 9.00 for Windows, GraphPad Software (La Jolla, CA, www.graphpad.com), and SPSS Software version 25 (Armonk, NY, www.ibm.com). A paired $t$-test was used to compare the means of the same group under two separate conditions. An unpaired or independent samples $t$ test was used to compare the means of two unrelated groups. The differences between group scores were considered statistically significant when $p<0.05$.

\section{RESULTS}

All mice successfully developed bilateral tumors and were subsequently divided into two experimental groups for local treatment: (i) Standard of care - Resection group, and (ii) Cryoablation group. The mean (SD) tumor volume for $\mathrm{T}_{\text {Baseline, }} \mathrm{Abs}_{\text {Res }}$, and $\mathrm{Abs}_{\text {Cryo }}$ was 279 (41) $\mathrm{mm}^{3}, 276$ (122) $\mathrm{mm}^{3}$, and $224(56) \mathrm{mm}^{3}$, respectively. There was no significant difference for tumor volume or weight between any of the three tumor groups. Cryoablated tumors remained in vivo and showed complete destruction by in vivo imaging (Fig. 2). For the cryoablation group, the average freeze/thaw/freeze cycles were 84/180/81 seconds. At 24-hour posttreatment assessment of the two local treatment procedures, no mice showed luciferase activity at the treatment site, indicating complete tumor ablation or tumor resection; however, the nontreated distant tumors still had a high fluorescent measurement (Fig. 2C).

One week after local treatment, the distant tumor was resected and labeled according to schema based on local therapy of primary tumors $\left(\mathrm{Abs}_{\mathrm{Res}}\right.$ or $\left.\mathrm{Abs}_{\mathrm{Cryo}}\right)$. All tumors were analyzed for TILs (Fig. 3). $\mathrm{T}_{\text {Baseline }}$ were remarkably consistent for overall TIL percentages. There was no correlation between TIL scores to tumor volume with all resected tumors having a stromal TIL score of $1 \%$ (Fig. 3B; Table 1). Both $\mathrm{Abs}_{\text {Res }}$ and $\mathrm{Abs}_{\text {Cryo }}$ had an overall increase in stromal and invasive margin TILs compared with $\mathrm{T}_{\text {Baseline. There was a significant increase in the mean (SD) }}$ stromal $(2.8[1.1] \% ; p=0.015)$ and invasive margin $(50$
[12.2]\%; $p=0.02)$ TILs for the Abs Cryo group compared with $\mathrm{T}_{\text {Baseline }}$ of 31 (4.9\%) (Fig. 3; Table 1). The $\mathrm{Abs}_{\text {Res }}$ did not show significant increase from $\mathrm{T}_{\text {Baseline }}$.

TILs were further assessed for changes in CTL markers in the $\mathrm{Abs}_{\text {Res }}$ and $\mathrm{Abs}_{\text {Cryo }}$ compared with $\mathrm{T}_{\text {Baseline by }}$ immunofluorescent staining for $\mathrm{CD} 3 \varepsilon$ ( $\mathrm{T}$ cells), CD8 $\alpha$ (CTLs), and granzyme B (activated CTLs) (Fig. 4). The $\mathrm{Abs}_{\text {Cryo }}$ tumors had an overall increase in CD3 $\varepsilon$ normalized fluorescence staining compared with both the $\mathrm{T}_{\text {Baseline }}$ and $\mathrm{Abs}_{\text {Res }}$ tumors. There was a slight increase in CD8 $\alpha$ staining in the $\mathrm{Abs}_{\text {Res }}$ group compared with $\mathrm{T}_{\text {Baseline, but }}$ virtually no change in $\mathrm{CD} 3 \varepsilon$ or granzyme $\mathrm{B}$ staining. It is noteworthy that in the $\mathrm{Abs}_{\mathrm{Cryo}}$ tumors, there was a significant increase in mean (SD) normalized fluorescence for CD8 $\alpha$ (15.7 [12.1]; $p=0.020$ ] and the CD8 CTL activation marker granzyme B (4.8 [3.6]; $p=0.048)$ compared with $\mathrm{T}_{\text {Baseline }}$ (5.2 [4.7] and 2.4 [0.09], respectively), indicating increased activated CTLs in the tumor (Fig. 4).

An evaluation comparing the standard of care group to the cryoablation group showed that cryoablation was superior to resection in preventing tumor recurrence (Fig. 5). At 4 weeks post-cryoablation, all mice were tumor-free by palpation and IVIS analysis. At 6 weeks post-cryoablation, two mice in the resection group had pinpoint tumors at the resection sites by palpation, which for one of the mice was easily detected by IVIS by 7 weeks (Fig. 5A). Two of five (40\%) mice in the resection group versus none of the five mice in the cryoablation group developed local and systemic failure of disease. Both tumor-positive mice had to be sacrificed due to humane endpoints, whereas the cryoablation group all appeared healthy and showed no signs of disease at sacrifice time points (Fig. 5B). Moreover, the cryoablation procedure showed minimal scar tissue with healthy mammary tissue at time of sacrifice (Fig. 5C). Of the mice in the resection group that developed tumor recurrence, one was at the initial resection-treated tumor site and the other was at the excised $\mathrm{Abs}_{\text {Res }}$ site (Fig. 5D; Table 1). Neither tumor had substantial number of TILs with $1 \%$ central tumor and less than $6 \%$ invasive margin TILs. Of note, two of the five mice in the resection group that did not mount a central tumor TIL response were the same mice that failed longterm control of tumor growth. Proportion test showed significant association between increase in TIL $\%$ and prevention of tumor recurrence $(p=0.02)$. Overall, TIL scores for the recurrent primary tumors was much lower than any of the previous tumor TIL scores analysis. The stroma TILs remained at $1 \%$, whereas the intratumor TILs decreased to $0 \%$ and the invasive margin TILs decreased to $3.5 \%$ and $5.5 \%$. Mice that developed tumor recurrence had increased spleen and liver weights (Fig. 5E) compared with the other mice with both mice developing splenomegaly (Fig. 5E) most likely due to tumor-induced granulopoiesis. 

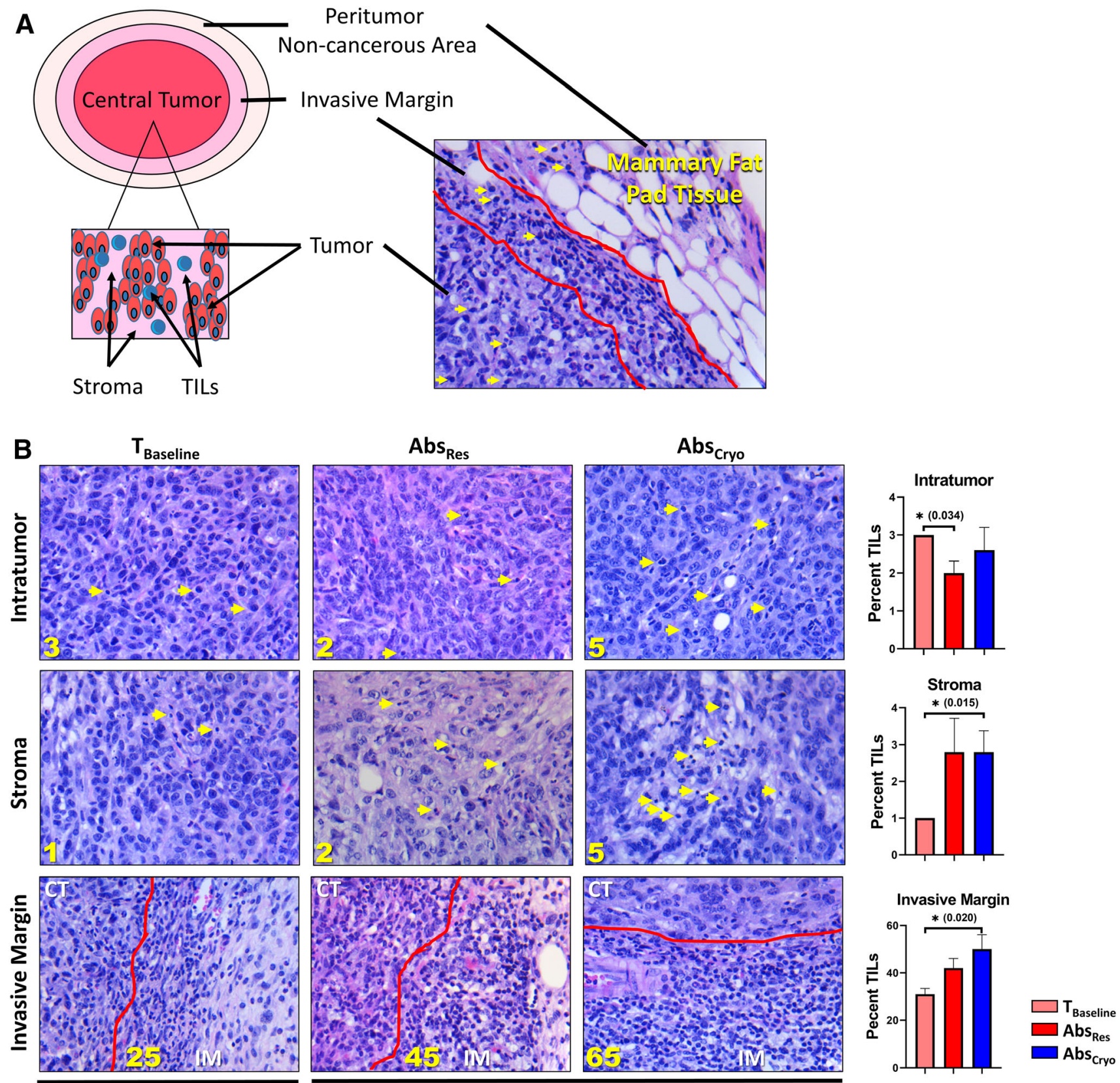

Local Tumor

Distant Tumor

FIG. 3 Tumor-infiltrating lymphocyte (TIL) scores. A Left, tumor schematic showing regions of lymphocytes in the tumor for TIL scoring; Right, H\&E histopathology demonstrating TILs in each region of the tumor used to score TILs. B Representative TIL analysis for the resected baseline control $\left(\mathrm{T}_{\mathrm{Baseline}}\right)$ and the distant tumor (Abs) from each treatment group showing a low, medium, and high TIL

Additionally, mice that had tumor recurrence exhibited metastasis to the lung (Fig. 5F) and suspicious regions in the liver (data not shown). These results indicate that local cryoablation therapy generated a strong systematic antitumor immune response and long-term antitumor immunity that protected against tumor recurrence and metastases. score. The tumors were analyzed for intratumor, stroma, and invasive margin TILs. Graphs on the far right are group mean with SD. There was a significant increase in stromal and invasive margin TILs for the $\mathrm{Abs}_{\text {Cryo }}$ group compared with baseline with significance considered $* p<0.05$

\section{DISCUSSION}

Among the hallmarks of cancer is a competition between the tumor attempting to escape immune surveillance and antitumor immunity versus the immune system recognizing and eliminating the tumor. ${ }^{28}$ This phenomenon is being extensively investigated in the local and systemic 
TABLE 1 Tumor characteristics and TIL scores

\begin{tabular}{|c|c|c|c|c|c|c|}
\hline \multicolumn{7}{|l|}{ TIL analysis } \\
\hline Mouse & Volume $\left(\mathrm{mm}^{3}\right)$ & Intratumor $(\%)$ & Stroma $(\%)$ & Invasive margin $(\%)$ & Tumor recurrence & Metastasis \\
\hline \multicolumn{7}{|c|}{ Baseline - resection treatment control group $\left(T_{\text {Baseline }}\right)$} \\
\hline M1 & 286 & 3 & 1 & 40 & N/A & \\
\hline M2 & 327 & 3 & 1 & 30 & & \\
\hline M3 & 317 & 3 & 1 & 30 & & \\
\hline M4 & 223 & 3 & 1 & 25 & & \\
\hline M5 & 240 & 3 & 1 & 30 & & \\
\hline Mean $\pm \mathrm{SD}$ & $279 \pm 41$ & $3 \pm 0.0$ & $1 \pm 0.0$ & $31 \pm 4.9$ & & \\
\hline \multicolumn{7}{|c|}{ Abscopal tumor - resection group $\left(A b s_{\text {Res }}\right)$} \\
\hline M1 & 502 & 2 & 5 & 40 & No & No \\
\hline M2 & 185 & 2 & 5 & 40 & No & No \\
\hline M3 & 218 & 1 & 1 & 55 & Abscopal site & Lung \\
\hline M4 & 303 & 2 & 2 & 45 & No & No \\
\hline M5 & 172 & 3 & 1 & 30 & Treatment site & Lung \\
\hline Mean \pm SD & $276 \pm 122$ & $2 \pm 0.6$ & $2.8 \pm 1.8$ & $42 \pm 8.1$ & $2 / 5(40 \%)$ & $2 / 5(40 \%)$ \\
\hline \multicolumn{7}{|c|}{ Abscopal tumor- cryoablation group $\left(A b s_{\text {Cryo }}\right)$} \\
\hline M6 & 303 & 5 & 5 & 65 & No tumor recurrence & \\
\hline M7 & 248 & 2 & 2 & 50 & No metastasis & \\
\hline M8 & 218 & 2 & 3 & 60 & & \\
\hline M9 & 223 & 2 & 2 & 30 & & \\
\hline M10 & 130 & 2 & 2 & 45 & & \\
\hline Mean \pm SD & $224 \pm 56$ & $2.6 \pm 1.2$ & $2.8 \pm 1.1$ & $50 \pm 12.2$ & $0 / 5(0 \%)$ & $0 / 5(0 \%)$ \\
\hline
\end{tabular}

environment created by the tumor and attempted therapies. Whereas local antitumor immunity is studied within the tumor microenvironment, the systemic response is a subject of investigation in terms of abscopal effect. The tumor microenvironment, comprised of immune cells, signaling molecules, extracellular matrix, adjacent blood vessels, and fibroblasts, is the battleground and has the potential to provide an advantage to one side, depending on its composition. Immune cells, in particular, play an essential role in this battle by providing the factors that comprise the tumor microenvironment-both adding to the antitumor defenses and blocking them. Even within lymphocytes, there are subtypes on either side. For example, tumor infiltration by $\mathrm{CD}^{+}$effector $\mathrm{T}$ cells is associated with significant antitumoral response and longer survival in breast carcinoma. ${ }^{13}$ Conversely, $\mathrm{T}_{\text {Reg }}$ cells, which normally suppress self-reactive $\mathrm{T}$ cells to fight autoimmune diseases, are believed to contribute to tumor tolerance by suppressing other immune cells. ${ }^{13}$ However, the overall percentage of lymphocytes in the tumor bed observed without subtyping are still predictive of better prognosis in TNBC and HER2 $^{+}$breast cancer. ${ }^{12,18-20}$
Breast cancer TIL score predicts the effectiveness of breast cancer treatment and prognosis. ${ }^{12,18-20}$ Breast cancer patients with a higher TIL score on diagnostic biopsy specimens appear to do better with adjuvant and neoadjuvant systemic therapy and result in less tumor metastasis. $^{12,18-20}$ The robust evidence supporting TIL scoring as an important prognostic marker led to the formation of the International Immuno-Oncology Biomarker Working Group on Breast Cancer that recommends regular TIL reporting on breast carcinomas following the guidelines provided in their 2017 report. $^{21}$ However, TIL scores have mainly been reported within the context of primary tumor. To the best of our knowledge, no studies have looked at the role of TIL scores in distant tumors as a potential biomarker of abscopal effect resulting from local therapy of primary tumor.

It is well established the systemic immune response plays the predominant role in executing the abscopal effect. $^{29}$ Previous studies have shown that primary tumors treated by ablation exerts a direct cytotoxic effect on the tumor cells and reprograms the tumor microenvironment. ${ }^{30}$ Tumor ablation initiates immunogenic cell death (ICD) releasing damage associated molecular pattern (DAMPs) molecules, cytokines, and chemokines, which recruit 


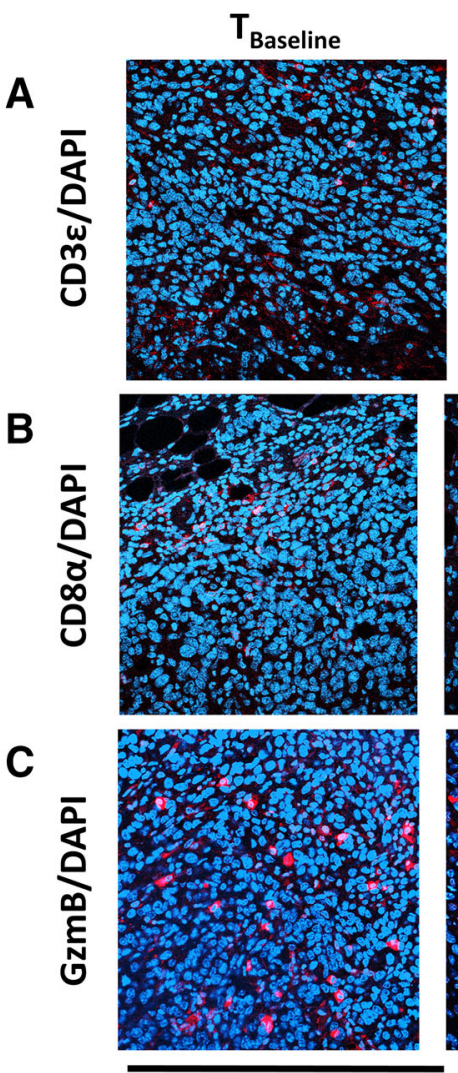

Treated
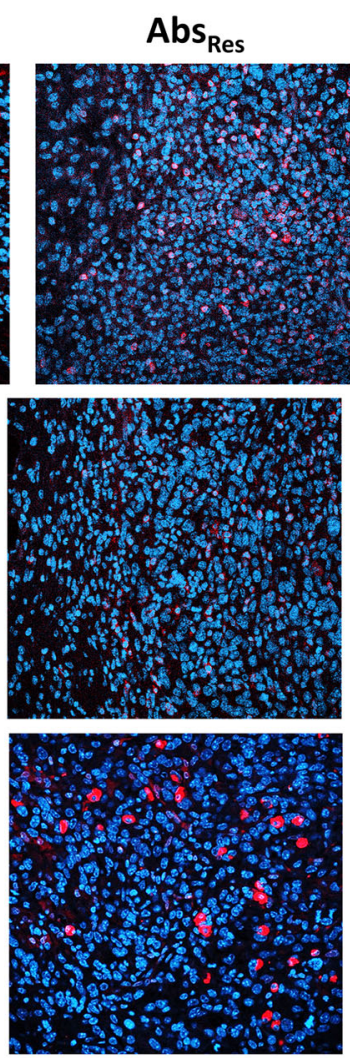

Readout

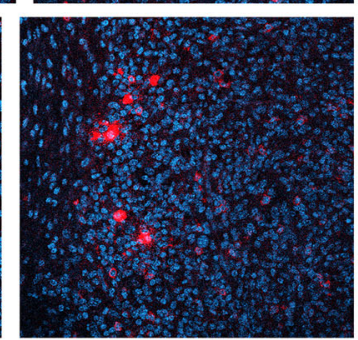

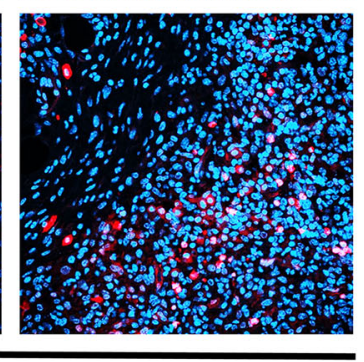
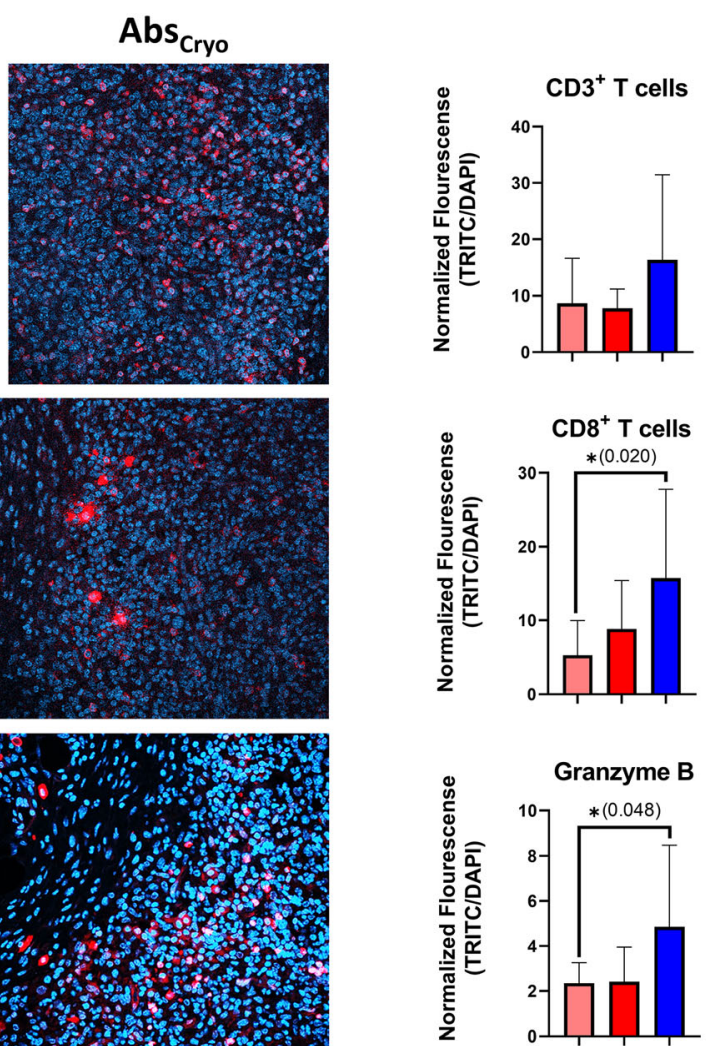

FIG. 4 TIL phenotyping for cytotoxic $\mathrm{T}$ lymphocytes. Immunofluorescent staining for $\mathrm{CD}^{+} \mathrm{T}$ cells, $\mathrm{CD} 8^{+} \mathrm{T}$ cells, and granzyme $\mathrm{B}$ - a cytotoxic $\mathrm{T}$-cell marker. Data shown are for $\mathrm{T}_{\text {Baseline, }}$ $\mathrm{Abs}_{\text {Res }}$, and $\mathrm{Abs}_{\text {Cryo }}$ tumors stained with $\mathbf{A}$ anti-CD3e (red), B anti$\mathrm{CD} 8 \propto$ (red), C anti-granzyme B (red) and DAPI (blue). Graphs on the

immune cells into the tumor and induces potent antitumor immune responses with increases in dendritic cell antigen presentation and activation of $\mathrm{CD}^{+} \mathrm{T}$ cells. ${ }^{31}$ These activated $\mathrm{CD}^{+} \mathrm{CTLs}$ then target the primary treated tumor and also can enter into the periphery as the prime driver of the abscopal effect targeting distant tumors and metastasis. ${ }^{32-34}$ Therefore, methodology to measure abscopal TILs theoretically could potentially serve as an innovative tool for monitoring and predicting cancer ablation treatment efficacy and long-term survival.

Cryoablation of breast cancer provides a viable alternative to traditional resection and thermal ablation where the tumor undergoes a series of freeze/thaw cycles but remains within the patient. ${ }^{35}$ As the "killed" tumor and surrounding tissue architecture is preserved, there remains the potential for augmentation of an immune response to the preserved TAAs (potentially measurable by TIL scoring), ${ }^{24}$ leading to an abscopal effect. This aligns with our findings of increased stromal and invasive margin TILs in the unmanipulated distant tumors 1 week after local therapy of primary tumor, with a significant increase for far right are group mean with SD for normalized fluorescence for each antibody marker. The cryoablation group shows increased CD3 $\varepsilon$ staining and a significant increase of $\mathrm{CD} 8 \alpha$ and granzyme B staining compared with baseline control tumors, with significance considered $* p<0.05$

stromal and invasive margin TILs in the cryoablation group. Moreover, we demonstrated that the TIL score of distant tumor correlates with systemic tumor burden and survival, albeit in a small sample. Thus, the distant, unmanipulated tumors represent a target to study abscopal effect resulting from the treatment of the first treated tumor using TIL score as a proxy measure for systemic disease control. Interestingly, we also found a lesser increase in TILs after resection of the first treated tumor, albeit not statistically significant. This modest effect could have resulted from the natural inflammatory response secondary to local trauma of handling and resection. Notably, 40\% (2/ 5) of the mice in the resected group developed both tumor recurrence and metastasis to the lung. The same mice were the only ones in the entire experiment (the resected and cryoablated groups combined) with no increase in stromal TILs compared with the baseline control tumors. This suggests that cryoablation provided a more robust antitumor response both locally and systemically, describing an 


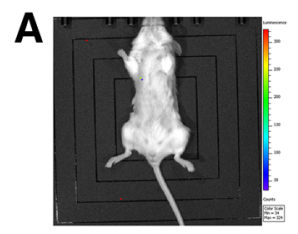

Week 4

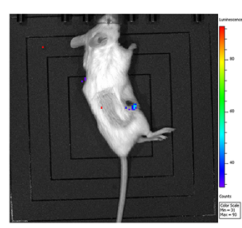

Week 7

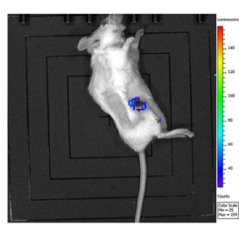

Week 8

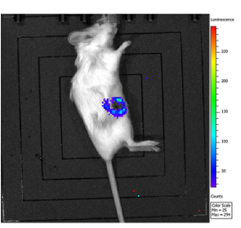

Week 9
B Survival Curve Comparing

Cryoablation vs. Resection Treated Mice

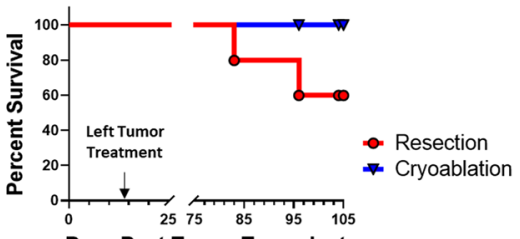

Days Post Tumor Transplant
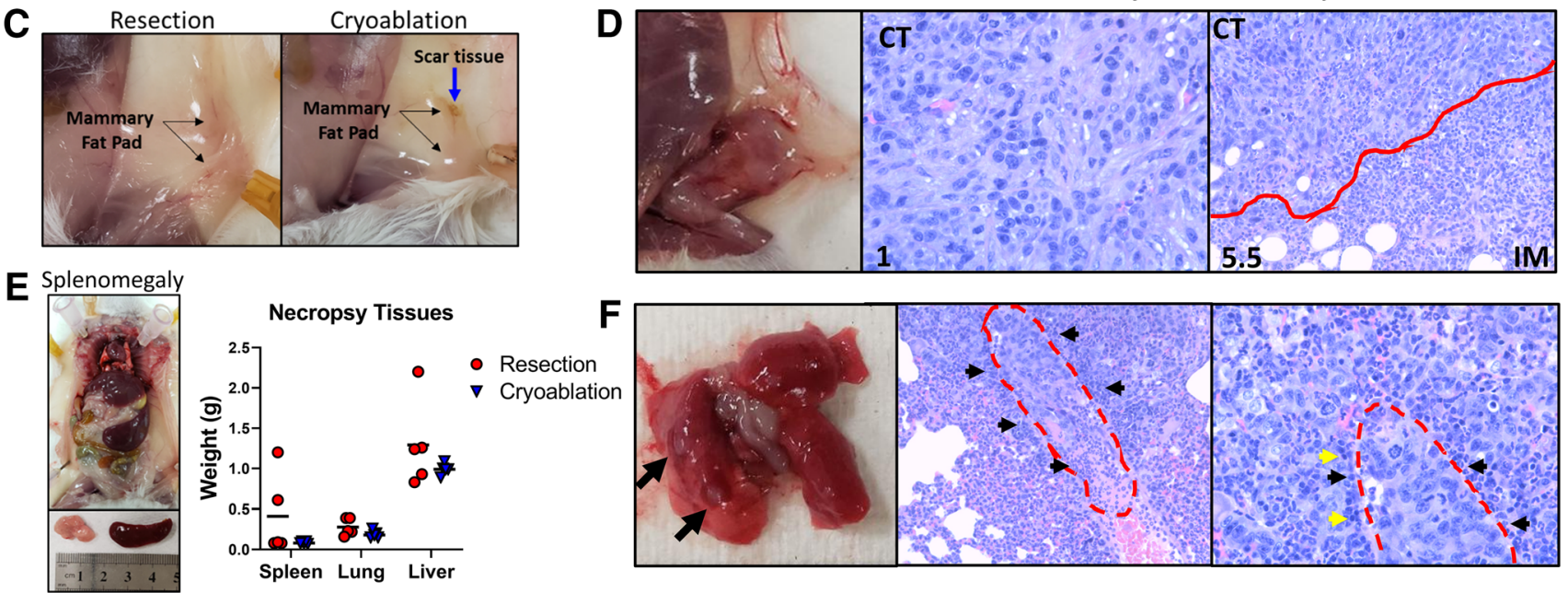

$\mathbf{F}$

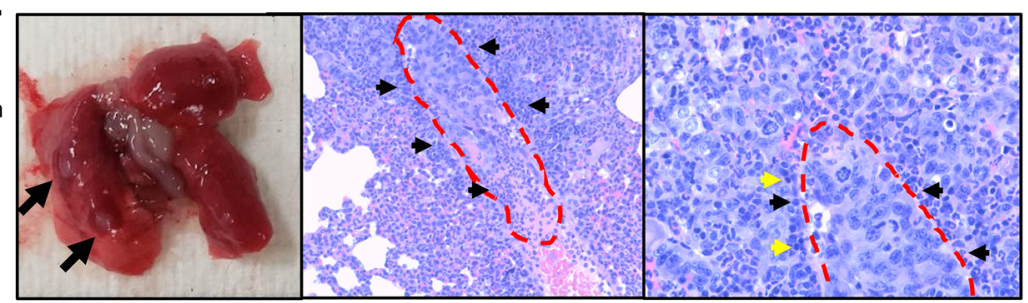

FIG. 5 Tumor recurrence and metastasis. A IVIS analysis showing primary tumor recurrence post-cryoablation. B Survival curves for resected vs. cryoablated mice. C Necropsy: Left, comparison of tumor site post-tumor treatment, the cryoablated tumor was completely reabsorbed leaving only small residual scar tissue. D Left, gross tissue showing primary tumor recurrence and right, H\&E analysis for TILs.

abscopal effect. More importantly, because the relapse was associated with lack of TIL response, TIL counts can be used as a proxy measure for abscopal effect.

While local resection, which is the mainstay for local control in the present time, removes the primary tumor and provides a minimal local inflammatory response, micrometastasis may still exist. More importantly, by removing the TAAs, resection denies an opportunity for the immune system to mount a robust response needed to overcome the potential for targeting metastasis. Cryoablation is clinically used for the treatment of fibroadenomas and low-risk cancers, ${ }^{35-39}$ mainly because of convincing evidence supporting complete destruction of tumors up to $15 \mathrm{~mm}$ in size. However, the potential of augmented immune response by virtue of leaving TAAs in vivo, is not very well-leveraged in the present day practice. This raises the concept of expanding cryoablation to high-risk tumors (TNBC and $\mathrm{HER}^{+}$) that are more immunogenic ${ }^{40}$ but have a poor prognosis to conventional treatments. Cryoablation may provide the immunologic boost needed for improved prognosis in these already immunogenic tumors.
E Gross tissue showing splenomegaly and graph of tissue weights for spleen, lung, and liver. F Left, lung metastasis nodules. Black arrows indicate tumors. Right, histopathology for lung metastasis. Red dash outlines metastatic tumor zone, black arrows indicate neutrophils and yellow arrows indicate TILs

Additionally, cryoablation could be combined with immune modulators to create a more robust immune response. Immune modulators target and inhibit checkpoint molecules like cytotoxic T lymphocyte antigen-4 (CTLA4) and programmed death-1/ligand-1 (PD-1/PD-L1). A 2016 pilot study found that the combination of ipilimumab, an immune modulator, with cryoablation in women with invasive breast cancer of any $\mathrm{HER}^{+}$and hormone receptor status led to peripheral elevations in Th1-type cytokines, activated and proliferating $\mathrm{CD}^{+}$and $\mathrm{CD}^{+} \mathrm{T}$ cells, and post-treatment proliferative T-effector cells relative to T-regulatory cells within the tumor. Study with a larger sample size focused on already immunogenic tumors could be performed to determine the potential benefit. ${ }^{41}$ As these studies on local therapies alone or in combination move forward in the clinical realm, it will be important to identify relevant and pragmatic biomarkers that could be utilized as outcome measures (indicative of impact on survival) to foster cost-effective clinical trials. This translational work is ideally suited for development of animal models that endorse biomarkers that could be successfully translated into clinical research and practice. 
Animal models for translational research provide insight into cancers and therapeutic responses that cannot be performed through clinical research studies. Although animal models have successfully helped to define mechanisms of cancer initiation, development, and treatment, there are clearly differences between rodent models and human cancer development. For example, breast cancer in humans tends to spread lymphatically to lymph nodes, then metastatically to the bones, brain, and adrenal gland, and then the liver and lung, whereas in the mouse, metastasis is almost exclusively through the hematogenous route to the lung and liver. ${ }^{42}$ Therefore, when utilizing animal models for cancer research, these differences should be considered for both analysis and interpretation of the results. For our study, we focused our metastasis analysis on the lung and liver; however, the immune system in both humans and mice is systemic and functions similarly. Therefore, correlations for the abscopal effect in the mouse can be extrapolated to humans. We found that metastasis was only observed in the mice that had tumor recurrence. It is possible that local tumor recurrence is a marker of systemic disease rather than the cause of metastasis. While immune cells were present at the site of metastases, they were primarily comprised of neutrophils, while lymphocytes, although present, were sparse (Fig. 5F). This also suggests that the surgical resection did not induce as strong of an antitumor CTL response in these mice to control tumor recurrence and metastasis. Most importantly, these outcomes were predictable in our model via TIL scoring of the abscopal readout tumors. Intuitively, the difference in TIL score from baseline to postablation in humans could potentially predict the desired abscopal effect.

Most previous breast cancer mouse models use a single tumor model, perform cryoablation or resection, and analyze the results for the immune response. ${ }^{33,34,43}$ Sabel et al. demonstrated that cryoablation of MT-901 tumors resulted in a tumor-specific immune response in the tumor draining lymph nodes, which correlated with rejections of tumors upon rechallenge. ${ }^{33}$ In our approach, we designed an experiment using two tumors to evaluate TILs in the distant tumor as an immunological readout to measure the abscopal effect. We found that changes in TIL scores in the abscopal-tumor appear to reflect whether mice developed recurrence and emphasize the importance of TILs for predicting long-term outcomes. Although a larger study is needed to confirm these results, this preliminary study reflects the utility of using TILs for measurement of abscopal effect.

Overall, we found that both resection and cryoablation induce an antitumor response with cryoablation being superior. It is likely that tumor resection results in both detrimental and beneficial effects on the systemic immune system, which might explain why $40 \%$ of the mice in the resection group failed to control disease. Surgery can trigger healing programs that lead to induce immunosuppressive states primarily driven by myeloid subsets, which can promote protumorigenic niches and suppress T-cell responses. Conversely, the benefit of resection with sufficient recovery time is reduced primary tumor burden allowing for restored systemic adaptive immune responses. ${ }^{44}$ We observed increased TILs in the distant tumors with a significant increase in stromal and invasive margin TILs for the cryoablation group. Furthermore, TIL phenotyping showed that the $\mathrm{Abs}_{\text {Cryo }}$ tumor TILs represent an increase in CTL markers by immunofluorescent staining. $\mathrm{CD}^{+} \mathrm{T}$ cells only express granzyme B following stimulation and differentiation into cytotoxic $\mathrm{T}$ cells. ${ }^{45}$ Remarkably, cryoablation induced a stronger abscopal CTL response with a significantly higher increase in both CD8 $\alpha$ and granzyme B staining when comparing the two treatment procedures to baseline tumors. We cannot attribute the granzyme $\mathrm{B}$ specifically to only $\mathrm{CD}^{+} \mathrm{T}$ cells, because activated $\mathrm{CD}^{+}$CTLs and NK cells also express granzyme B. However, the data show an increase in immune cytolytic activity in the $\mathrm{Abs}_{\mathrm{Cryo}}$ tumors. Interestingly, only mice that did not have an increase in stromal TILs posttreatment in the resection group had recurrence and metastasis, suggesting that induced TIL response is important in predicting overall disease control. The TIL score and CTL analysis in combination with the lack of tumors posttreatment showed that cryoablation resulted in an overall greater abscopal effect in preventing tumor recurrence and metastasis. Because the tumor microenvironment influences the TIL response, it will be important to further characterize the TILs for both antitumor and suppressive phenotypes and quantitate the ratios of activated CTLs $\left(\mathrm{CD}^{+} \mathrm{ICOS}^{++}\right)$versus $\mathrm{T}_{\mathrm{Regs}}\left(\mathrm{CD} 4^{+} \mathrm{FOXP}^{+}\right)$ posttreatment. These ratios could potentially be used as an additional TIL scoring method for predicted treatment efficacy for cryoablation or novel therapies. Future experiments will focus on using RNA-seq analysis to detect "immune gene signature" differences between resection and cryoablation using gene expression levels of immunerelated genes to describe the composition and functional status of the immune cell infiltrates, which could be translated for clinical applications.

Questions remain about why some individuals immunologically respond to treatment better than others and how to enhance the response in poor responders. These could be helped by studying the baseline TIL responses mounted by individuals and the ability of local therapies to enhance this response. 


\section{CONCLUSIONS}

Primary local treatment of triple-negative breast cancer by both resection and cryoablation increases systemic immune response, albeit the response is superior with cryoablation. Unmanipulated tumor TIL scoring provides a viable biomarker for studying the abscopal effect of local therapies.

ACKNOWLEDGMENTS The authors thank ASCO Equipment for the endowment for Excellence in Women's Health that provided financial support, Sanarus Technologies Inc. for the donation of cryoablation probes, and the TTUHSC LARC staff for assisting with animal care and veterinary consulting during the course of experiments. Fluorescent images were generated in the Image Analysis Core Facility supported in part by TTUHSC.

DISCLOSURE The author(s) declared no potential conflict of interest with reference to the research, authorship, and/or publication of this article.

OPEN ACCESS This article is licensed under a Creative Commons Attribution 4.0 International License, which permits use, sharing, adaptation, distribution and reproduction in any medium or format, as long as you give appropriate credit to the original author(s) and the source, provide a link to the Creative Commons licence, and indicate if changes were made. The images or other third party material in this article are included in the article's Creative Commons licence, unless indicated otherwise in a credit line to the material. If material is not included in the article's Creative Commons licence and your intended use is not permitted by statutory regulation or exceeds the permitted use, you will need to obtain permission directly from the copyright holder. To view a copy of this licence, visit http://creativecommons. org/licenses/by/4.0/.

\section{REFERENCES}

1. Mole RH. Whole body irradiation; radiobiology or medicine? $\mathrm{Br}$ J Radiol. 1953;26(305):234-41.

2. Fend L, Yamazaki T, Remy C, et al. Immune checkpoint blockade, immunogenic chemotherapy or IFN-alpha blockade boost the local and abscopal effects of oncolytic virotherapy. Cancer Res. 2017;77(15):4146-57.

3. Qu S, Worlikar T, Felsted AE, et al. Non-thermal histotripsy tumor ablation promotes abscopal immune responses that enhance cancer immunotherapy. $J$ Immunother Cancer. 2020;8(1).

4. Takaki H, Cornelis F, Kako Y, Kobayashi K, Kamikonya N, Yamakado K. Thermal ablation and immunomodulation: From preclinical experiments to clinical trials. Diagn Interv Imaging. 2017;98(9):651-9.

5. Aarts BM, Klompenhouwer EG, Rice SL, et al. Cryoablation and immunotherapy: an overview of evidence on its synergy. Insights Imaging. 2019;10(1):53.

6. Abuodeh Y, Venkat P, Kim S. Systematic review of case reports on the abscopal effect. Curr Probl Cancer. 2016;40(1):25-37.

7. Ozpiskin OM, Zhang L, Li JJ. Immune targets in the tumor microenvironment treated by radiotherapy. Theranostics. 2019;9(5):1215-31.
8. Grass GD, Krishna N, Kim S. The immune mechanisms of abscopal effect in radiation therapy. Curr Probl Cancer. 2016;40(1):10-24.

9. Dieu-Nosjean MC, Antoine M, Danel C, et al. Long-term survival for patients with non-small-cell lung cancer with intratumoral lymphoid structures. J Clin Oncol. 2008;26(27):4410-7.

10. Pages F, Kirilovsky A, Mlecnik B, et al. In situ cytotoxic and memory $\mathrm{T}$ cells predict outcome in patients with early-stage colorectal cancer. J Clin Oncol. 2009;27(35):5944-51.

11. Hwang WT, Adams SF, Tahirovic E, Hagemann IS, Coukos G. Prognostic significance of tumor-infiltrating $\mathrm{T}$ cells in ovarian cancer: a meta-analysis. Gynecol Oncol. 2012;124(2):192-8.

12. Denkert C, Loibl S, Noske A, et al. Tumor-associated lymphocytes as an independent predictor of response to neoadjuvant chemotherapy in breast cancer. J Clin Oncol. 2010;28(1):105-13.

13. Mahmoud SM, Paish EC, Powe DG, et al. Tumor-infiltrating CD8+ lymphocytes predict clinical outcome in breast cancer. $J$ Clin Oncol. 2011;29(15):1949-55.

14. Seo AN, Lee HJ, Kim EJ, et al. Tumour-infiltrating CD8+ lymphocytes as an independent predictive factor for pathological complete response to primary systemic therapy in breast cancer. Br J Cancer. 2013;109(10):2705-13.

15. Teschendorff AE, Gomez S, Arenas A, et al. Improved prognostic classification of breast cancer defined by antagonistic activation patterns of immune response pathway modules. BMC Cancer. 2010;10:604.

16. Mahmoud SM, Lee AH, Paish EC, Macmillan RD, Ellis IO, Green AR. The prognostic significance of B lymphocytes in invasive carcinoma of the breast. Breast Cancer Res Treatment. 2012;132(2):545-53.

17. Salgado R, Denkert C, Demaria S, et al. The evaluation of tumorinfiltrating lymphocytes (TILs) in breast cancer: recommendations by an International TILs Working Group 2014. Ann Oncol. 2015;26(2):259-71.

18. Loi S, Sirtaine N, Piette F, et al. Prognostic and predictive value of tumor-infiltrating lymphocytes in a phase III randomized adjuvant breast cancer trial in node-positive breast cancer comparing the addition of docetaxel to doxorubicin with doxorubicinbased chemotherapy: BIG 02-98. J Clin Oncol. 2013;31(7):860-7.

19. Loi S, Michiels S, Salgado R, et al. Tumor infiltrating lymphocytes are prognostic in triple negative breast cancer and predictive for trastuzumab benefit in early breast cancer: results from the FinHER trial. Ann Oncol. 2014;25(8):1544-50.

20. Adams S, Gray RJ, Demaria S, et al. Prognostic value of tumorinfiltrating lymphocytes in triple-negative breast cancers from two phase III randomized adjuvant breast cancer trials: ECOG 2197 and ECOG 1199. J Clin Oncol. 2014;32(27):2959-66.

21. Dieci MV, Radosevic-Robin N, Fineberg S, et al. Update on tumor-infiltrating lymphocytes (TILs) in breast cancer, including recommendations to assess TILs in residual disease after neoadjuvant therapy and in carcinoma in situ: a report of the International Immuno-Oncology Biomarker Working Group on Breast Cancer. Semin Cancer Biol. 2018;52(Pt 2):16-25.

22. Denkert C, von Minckwitz G, Darb-Esfahani S, et al. Tumourinfiltrating lymphocytes and prognosis in different subtypes of breast cancer: a pooled analysis of 3771 patients treated with neoadjuvant therapy. Lancet Oncol. 2018;19(1):40-50.

23. $\mathrm{Ng} \mathrm{KK}$, Lam CM, Poon RT, et al. Comparison of systemic responses of radiofrequency ablation, cryotherapy, and surgical resection in a porcine liver model. Ann Surg Oncol. 2004;11(7):650-7.

24. Lees JS, Dooley WC. Nonsurgical ablation of primary breast cancer. Surg Oncol Clin N Am. 2005;14(1):33-44. 
25. Kumar AV, Patterson SG, Plaza MJ. Abscopal Effect following Cryoablation of Breast Cancer. $J$ Vasc Interv Radiol. 2019;30(3):466-9.

26. Tao K, Fang M, Alroy J, Sahagian GG. Imagable 4T1 model for the study of late stage breast cancer. BMC Cancer. 2008;8:228.

27. Yosofvand M, Liyanage S, Kalupahana NS, Scoggin S, Moustaid-Moussa N, Moussa $\mathrm{H}$. AdipoGauge software for analysis of biological microscopic images. Adipocyte. 2020;9(1):360-73. https://doi.org/10.1080/21623945.2020.1787583

28. Fouad YA, Aanei C. Revisiting the hallmarks of cancer. Am $J$ Cancer Res. 2017;7(5):1016-36.

29. Tranberg KG. Local destruction of tumors and systemic immune effects. Front Oncol. 2021;11:708810.

30. Weichselbaum RR, Liang H, Deng L, Fu YX. Radiotherapy and immunotherapy: a beneficial liaison? Nat Rev Clin Oncol. 2017;14(6):365-79.

31. Zhou J, Wang G, Chen Y, Wang H, Hua Y, Cai Z. Immunogenic cell death in cancer therapy: present and emerging inducers. $J$ Cell Mol Med. 2019;23(8):4854-65.

32. Lee Y, Auh SL, Wang Y, et al. Therapeutic effects of ablative radiation on local tumor require $\mathrm{CD} 8+\mathrm{T}$ cells: changing strategies for cancer treatment. Blood. 2009;114(3):589-95.

33. Sabel MS, Nehs MA, Su G, Lowler KP, Ferrara JL, Chang AE. Immunologic response to cryoablation of breast cancer. Breast Cancer Res Treatment. 2005;90(1):97-104.

34. Sabel MS, Su G, Griffith KA, Chang AE. Rate of freeze alters the immunologic response after cryoablation of breast cancer. Ann Surg Oncol. 2010;17(4):1187-93.

35. Habrawi Z, Melkus MW, Khan S, et al. Cryoablation: a promising non-operative therapy for low-risk breast cancer. Am J Surg. 2021;221(1):127-33.

36. Niu L, Wu B, Xu K. Cryosurgery for breast fibroadenomas. Gland Surg. 2012;1(2):128-31.
37. Kaufman CS, Littrup PJ, Freeman-Gibb LA, et al. Office-based cryoablation of breast fibroadenomas with long-term follow-up. Breast J. 2005;11(5):344-50.

38. Littrup PJ, Jallad B, Chandiwala-Mody P, D'Agostini M, Adam BA, Bouwman D. Cryotherapy for breast cancer: a feasibility study without excision. $J$ Vasc Interv Radiol. 2009;20(10):1329-41.

39. Paepke S, Metz S, Brea Salvago A, Ohlinger R. Benign Breast tumours - diagnosis and management. Breast Care (Basel Switz). 2018;13(6):403-12.

40. Prat A, Pineda E, Adamo B, et al. Clinical implications of the intrinsic molecular subtypes of breast cancer. Breast. 2015;24(Suppl 2):S26-35.

41. McArthur HL, Diab A, Page DB, et al. A pilot study of preoperative single-dose ipilimumab and/or cryoablation in women with early-stage breast cancer with comprehensive immune profiling. Clin Cancer Res. 2016;22(23):5729-37.

42. Cheung A, Young L, Chen $\mathrm{P}$, et al. Microcirculation and metastasis in a new mouse mammary tumor model system. Int $J$ Oncol. 1997;11(1):69-77.

43. Zhu J, Zhang Y, Zhang A, He K, Liu P, Xu LX. Cryo-thermal therapy elicits potent anti-tumor immunity by inducing extracellular Hsp70-dependent MDSC differentiation. Sci Rep. 2016;6:27136.

44. Hiam-Galvez KJ, Allen BM, Spitzer MH. Systemic immunity in cancer. Nat Rev Cancer. 2021;21(6):345-59.

45. Nowacki TM, Kuerten S, Zhang W, et al. Granzyme B production distinguishes recently activated $\mathrm{CD} 8(+)$ memory cells from resting memory cells. Cell Immunol. 2007;247(1):36-48.

Publisher's Note Springer Nature remains neutral with regard to jurisdictional claims in published maps and institutional affiliations. 